\title{
Nonstereotyped Responding in Positive Schizotypy after a Single Dose of Levodopa
}

\author{
Christine Mohr* ${ }^{*, 1,4,5}$, Theodor Landis², Peter S Sandor ${ }^{5}$, Marc Fathi ${ }^{3}$ and Peter Brugger ${ }^{5}$ \\ 'Functional Brain Mapping Laboratory, University Hospital Geneva, Geneva, Switzerland; ²Neurology Clinic, University Hospital Geneva, Geneva, \\ Switzerland; ${ }^{3}$ Clinical Chemistry Central Laboratory, University Hospital Geneva, Geneva, Switzerland; ${ }^{4}$ Rehabilitation Clinic, University Hospital \\ Geneva, Geneva, Switzerland; ${ }^{5}$ Neurology Clinic, University Hospital Zurich, Zurich, Switzerland
}

\begin{abstract}
Stereotyped behavior and left-sided orientation biases, associated with the dopamine (DA) system, were observed in populations of the schizophrenia spectrum disorders. We investigated whether heightened DA concentrations influence both side biases and stereotyped responding in a visuo-motor computer task, in which 90,180 , and $270^{\circ}$ rotated objects had to be brought into a target position. To account for the role of the schizophrenia spectrum, task performance was also analyzed as a function of healthy participants' high or low magical ideation (MI), a positive schizotypal feature. The first 36 participants (20 women) remained substance free. In a second sample, 20 men received levodopa and 20 men a placebo in a double-blind procedure. Results showed that high Ml scorers responded more stereotyped than low Ml scorers, without being specifically biased towards the left side. Rotation preferences toward one or the other side made high $\mathrm{Ml}$ scorers less flexible for objects efficiently to be rotated into the opposite direction. This inflexibility may reflect impaired left hemisphere functioning. Unexpectedly, in the levodopa group, high Ml scorers performed superior to low Ml scorers. Since DA actions appear to follow an inverted U-shape function, the 'low' performing high Ml scorers profited from the enhanced DA availability. Our observation in the levodopa group points to a dissociation between schizotypy and schizophrenia: while cognitive improvement in schizophrenia can occur after treatment with atypical neuroleptic agents, in our positive schizotypal participants a DA agonist resulted in improved task performance. This dissociation may point to protective neurochemical mechanisms preventing healthy schizotypes from developing full-blown psychotic symptoms.

Neuropsychopharmacology (2004) 29, I74|-|75I, advance online publication, 2 June 2004; doi: I 0. I038/sj.npp. 1300500
\end{abstract}

Keywords: dopamine; turning behavior; stereotypy; perseveration; positive symptoms

\section{INTRODUCTION}

Dopamine (DA) and the emergence of psychosis are incontestably tied. Patients with schizophrenia profit from DA antagonistic treatment (Laruelle and Abi-Dargham, 1999) and show worsening of psychotic symptoms after DA agonistic treatment (Abi-Dargham et al, 1998; Davidson et al, 1987). In healthy populations, DA agonists have the potential to trigger psychotic symptoms (Janowsky and Risch, 1979; Sekine et al, 2001). Consequently, acute psychotic symptoms were assumed to emerge from a hyperactive DA system (Davis et al, 1991; Laruelle and Abi-Dargham, 1999).

\footnotetext{
*Correspondence: Dr C Mohr, Functional Brain Mapping Laboratory, Neurology Clinic, University Hospital Geneva, Rue Micheli-du-Crest 24, CH-I2II Geneva 14, Switzerland, Fax: +4I 22372 8358,

E-mail: Christine.Mohr@hcuge.ch

Received 22 December 2003; revised 24 March 2004; accepted 27 April 2004

Online publication: 4 May 2004 at http://www.acnp.org/citations/ Npp05040403570/default.pdf
}

Among the behavioral markers for a hyperactive DA system are stereotyped movements. As known from the animal literature, the administration of DA agonists can trigger stereotyped behavior (Staton and Solomon, 1984; Kelley et al, 1988; Kuczenski and Segal, 1999), particularly after high doses (Randrup and Munkvad, 1974). It is thus not a surprise that stereotyped behavior is observed in patients with schizophrenia (Kraeplin, 1919; Bleuler, 1911/ 1950; Jones, 1965; Peralta and Cuesta, 2001) and in healthy persons after amphetamine abuse (Connell, 1958, Ridley et al, 1988). Rocking, walking backwards and forwards, biting and other repetitive movements of the jaw, part of limbs, or even whole limbs were described as stereotyped behavior in schizophrenia (Jones, 1965). Stereotyped behavior also occurs at the cognitive level (see also Garner et $a l, 2003)$ in various facets along the schizophrenia spectrum. For instance, perseverative errors in the Wisconsin Card Sorting Test (WCST) are elevated in patients with schizophrenia (Perry and Braff, 1998 for overview), schizotypal personality disorder (Raine et al, 1992; Cadenhead et al, 1999) and healthy schizotypal participants (Lenzenweger and Korfine, 1994; Gooding et al, 1999). 
During the generation of random sequences, patients with schizophrenia (Salamé et al, 1998; Brugger, 1997 for overview), healthy participants after amphetamine administration (Ridley et al, 1988), and healthy participants with elevated schizotypal features (Brugger et al, 1990; Avons et al, 2003) showed more pronounced response biases compared to their respective controls.

Another behavioral marker for a hyperactive DA system is turning behavior, which depicts a relatively hyperactive DA system in one over the other hemisphere. Well known from the animal literature (Pycock, 1983 for overview) and from one study with patients with asymmetrical Parkinson's disease (Bracha et al, 1987), the preferred side of wholebody turning occurs away from the hemisphere with the more active DA system. Supporting the notion of a relative hyperactivation of the right-hemispheric DA system, acutely psychotic patients (Bracha et al, 1993) and healthy participants with positive schizotypal features (Mohr et al, 2003a) displayed a significant preference for left- over rightsided turns.

From the previous sections, it can be concluded that stereotyped movements and turning behavior seem to be promising behavioral markers to depict the activity of the DA system. Stereotyped behavior and left-sided turning biases along the schizophrenia spectrum suggest a (dysfunctional) hyperactive DA system relevant to all kinds of psychotic(-like) thought. However, it is not known how far nonspatial and spatial (turning) stereotyped behaviors in the schizophrenia spectrum are mediated by common dopaminergic actions. As noted above, both measures are enhanced under a presumably hyperdopaminergic state and attenuated with DA antagonistic treatment, in particular with second-generation antipsychotic medication (Levine et al, 1997; Kuczenski and Segal, 1999; Purdon et al, 2001; Harvey et al, 2003). Since the previous observations would suggest that the two measures are somehow interrelated, we determined the modification of both measures by a potentially hyperdopaminergic state in two experiments. In both experiments, the relative strength of hyperdopaminergia was inferred from individuals' positive schizotypal features, that is, magical ideation (MI, Eckblad and Chapman, 1983). In Experiment 2, participants' dopaminergic state was directly manipulated by levodopa administration.

In both experiments, we assessed nonspatial and spatial (turning) response stereotypies in a computer task (see Figure 1) resembling the popular TETRIS game. Objects 'dropped' down on the screen and had to be rotated (by 90 , 180 , or $270^{\circ}$ ) to match a target orientation indicated at the bottom of the computer screen (see Figure 1). In total, $2 \mathrm{~s}$ were allotted to accomplish one trial. By counting the number of key presses eliciting either a $90^{\circ}$ left-sided or a $90^{\circ}$ right-sided rotation, we could determine rotational preferences to either side. Critically, for target orientations rotated $180^{\circ}$ relative to the dropping stimulus, left- or rightsided rotations would be equally efficient (two subsequent key presses in any case), but one side might be favored over the other. Moreover, in case of stereotyped responding towards one side, task performance would be most efficient for some stimuli, but inferior for other. For instance, stereotyped left-sided rotation preferences would facilitate task efficiency for $90^{\circ}$ rotated objects (one left-sided key

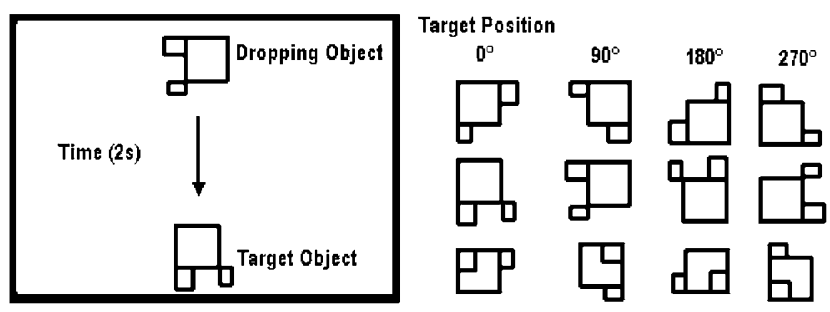

Figure I Sample trial of the computer task (left panel). Note that one left-sided key press or three right-sided key presses during $2 \mathrm{~s}$ would rotate the dropping object into the target position as indicated at the bottom of the screen. The right panel of the figure depicts the three different target positions used as well as the respective dropping objects in three different orientations each.

press), but would be disadvantageous for $270^{\circ}$ rotated objects (three left-sided key presses instead of one rightsided key press). Under the assumption that rotations in a $2 \mathrm{D}$ plane are modulated by the DA system in a way comparable to those of one's body in 3D space, we predicted for our participants without levodopa supplementation an association between left-sided rotation preferences and elevated MI scores (Mohr et al, 2003a). In case of stereotyped responding, this left-sided rotation preference should result in an efficient rotation strategy for $90^{\circ}$ objects, but an inefficient rotation strategy for $270^{\circ}$ objects. DA agonists can trigger psychotic symptoms in healthy persons (Janowsky and Risch, 1979; Sekine et al, 2001) and worsen psychotic symptoms in patients (Davidson et al, 1987; AbiDargham et al, 1998). Thus, in case of a similar modulation of the two behavioral measures by the DA system, we would expect that participants, in particular those with high MI scores, would show an even stronger left-sided rotation preference and more stereotyped left-sided key presses (specifically evident for $270^{\circ}$ objects) after levodopa as compared to placebo supplementation.

The use of the schizotypy approach in the present study is advantageous for several reasons. First, it would be unethical to provide DA agonistic substances to patients with schizophrenia, given their potential to worsen psychotic symptoms (Davidson et al, 1987; Abi-Dargham et al, 1998). Second, this line of research became well accepted to study brain functions related to schizophrenia free from confounding correlates of severe clinical symptoms such as medication and institutionalization (eg Gooding et al, 1999; Avons et al, 2003). In fact, preselected and unselected schizotypal individuals, thought to be 'psychosis-prone' (Chapman et al, 1994; Kwapil et al, 1997), perform similar to patients with schizophrenia for cognitive (Gooding et al, 1999; Park, 1999), attentional (Sarkin et al, 1998; Mohr et al, 2003a), behavioral (Barnett and Corballis, 2002; Mohr et al, 2003b) and physiological (Klein et al, 1999; Pizzagalli et al, 2000) measures. Finally, alterations of dopaminergic responsivity may accompany the schizophrenia spectrum disorders in a continuous manner; the DA metabolite homovanillin acid (HVA) correlated with severity of positive symptoms in schizophrenia (Pickar et al, 1984; Davidson and Davis, 1988) and the extent of schizotypal personality disorder (Siever et al, 1991, 1993). 


\section{EXPERIMENT 1}

\section{Participants}

A total of 36 healthy participants ( 20 women) were recruited by flyers. Volunteers with any current medication or a history of drug abuse or neuropsychiatric illnesses had been excluded. The relevant screening followed the guidelines offered by Campbell (2000). All participants were righthanded according to a 13-item handedness questionnaire (Chapman and Chapman, 1987). The local ethics committee had approved the study, and after complete description of the study to the participants, written informed consent was obtained.

\section{Magical Ideation Scale}

Each participant filled in a validated 30-item questionnaire that includes items such as 'I sometimes have a feeling of gaining or losing energy when people look at me or touch me,' (keyed true) or 'Some people can make me aware of them just by thinking about me' (keyed true). Scores on the MI scale range from 0 to 30 , with higher scores indicating more pronounced magical thinking. The scale is published in full in Eckblad and Chapman (1983) and in Barnett and Corballis (2002), and normative data can be found in Garety and Wessely (1994).

\section{Visuo-Motor Rotation Task}

Stimuli. Three different line drawings were composed from the same elements (one square, two rectangles; see Figure 1). The elements appeared on the computer screen in the following sizes: square: $10 \mathrm{~mm} \times 10 \mathrm{~mm}$, larger rectangle: $4 \mathrm{~mm} \times 5 \mathrm{~mm}$, and smaller rectangle: $4 \mathrm{~mm} \times 3 \mathrm{~mm}$.

Procedure. The line drawings appeared one after the other at the top of a computer screen ( $18 \mathrm{~cm}$ height; $25 \mathrm{~cm}$ width). Each of them appeared for $2 \mathrm{~s}$. During this time period, the drawings 'fell down' continuously in a straight line toward a target object presented at the bottom of the screen (Figure 1, left). The target object was identical to the dropping object, but the dropping object appeared either in the same orientation $\left(0^{\circ}\right)$, or $90^{\circ}, 180^{\circ}$, or $270^{\circ}$ rotated from the target object (Figure 1, right). Each line drawing was presented nine times. Thus, there were 27 stimulations per object orientation. The participant was instructed to turn the object as fast and accurately as possible into the target position by pressing either a key for $90^{\circ}$ counterclockwise (button: $\leftarrow$ ) or another key for $90^{\circ}$ clockwise (button: $\rightarrow$ ) rotations. For instance, to match a $180^{\circ}$ object with the target position, participants could rotate the object by two presses of the same key (either in the clockwise or counterclockwise direction). Participants could also complete a trial by pressing another key (button: $\downarrow$ ). On pressing this key, the object dropped down immediately. The whole experiment of 108 trials was conducted twice, once with the right hand and once with the left hand. Half of the participants started with the left, the other half with the right hand. The number of key presses to the left and right, respectively, were summed for each object orientation and across the two performing hands.

\section{Data Analysis}

Despite the fact that participants were explicitly instructed to rotate the objects in the fastest and most accurate way, two participants did not obey to these instructions and were excluded from the analyses. One participant made use of the left-sided key only nine times (MI score $=4$ ), while the remaining participants made on average $149.9 \pm 68.8$ leftsided key presses. The other participant made only 27 correct trials across blocks (MI score $=12$ ), while the remaining participants made at least 139 correct responses $(182.3 \pm 21.4)$. The remaining group of 34 participants was split at the median MI scale score (9) into a high $(n=18)$ and low $(n=16)$ MI group.

To obtain a measure indicating rotational preferences to the left or right side, we calculated a conventional laterality index score (number of left-sided key presses minus number of right-sided key presses divided by the sum of all key presses; Marshall et al, 1975) for $90^{\circ}, 180^{\circ}$ and $270^{\circ}$ objects separately. Consequently, positive values indicate a left-sided rotation preference and negative values a rightsided rotation preference. A three-way ANOVA with MI groups (high $v s$ low) and gender (men $v s$ women) as between-subject measure was performed on the laterality index scores for $90^{\circ}, 180^{\circ}$, and $270^{\circ}$ objects as repeated measure. To test whether rotational biases were associated with task efficiency (accuracy), we calculated the same index scores as described above, but only for correct responses. We performed an analogous ANOVA as described above, but on the laterality index scores for correct trials. Post hoc single comparisons were assessed with Duncan's multiple range tests correcting for multiple comparisons. All tests were two-tailed and the alpha-level was set at 0.05 .

Table I Descriptive Data of the Study Sample of Experiment I, for the Two Magical Ideation (MI) Groups and Women and Men Separately

\begin{tabular}{lrrrrr}
\hline & \multicolumn{2}{c}{ Low MI group } & & \multicolumn{2}{c}{ High MI group } \\
\cline { 2 - 5 } & Women & Men & Women & Men \\
\hline Age (years) & $31.4 \pm 7.3$ & $28.8 \pm 5.4$ & $27.3 \pm 4.3$ & $30.0 \pm 7.2$ \\
Education (years) & $21.0 \pm 4.2$ & $18.5 \pm 3.4$ & $18.8 \pm 3.3$ & $18.3 \pm 3.3$ \\
Ml scores & $4.6 \pm 2.6$ & $5.1 \pm 3.0$ & $14.5 \pm 5.8$ & $12.3 \pm 2.4$ \\
\hline
\end{tabular}


Table 2 Mean $( \pm \mathrm{SD})$ Laterality Index Score for $90^{\circ}, 180^{\circ}$, and $270^{\circ}$ Rotated Objects for the Whole Sample as well as for the Gender Groups Separately

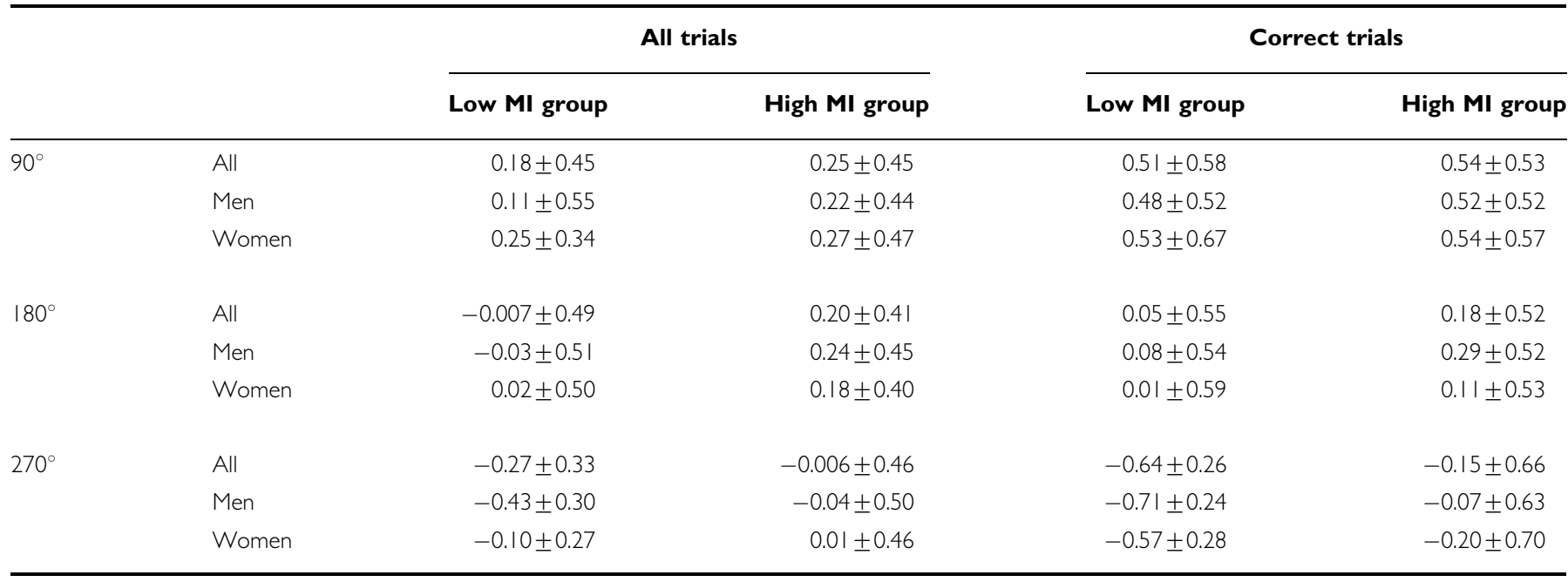

Values are presented for the two magical ideation (MI) groups for all trials (correct+incorrect) and correct trials, only.

\section{RESULTS AND BRIEF DISCUSSION}

\section{Participants}

Separate three-way ANOVAs with MI groups (high vs low) and gender (women $v s$ men) on (1) age, and (2) years of education were not significant (all F-values $<1.7$, $\mathrm{df}=1,30$; all $p$-values $>0.20$; Table 1). A comparable ANOVA on MI scores ruled out any gender difference with respect to the MI scores in the two MI groups, since there was neither a main effect for gender $(\mathrm{F}(1,30)=0.36, p=0.55)$ nor an interaction between gender and MI group $(F(1,30)=0.91$, $p=0.35$; Table 1 ).

\section{Visuo-Motor Rotation Task}

Laterality index scores for all trials, irrespective of correctness (see Table 2): The ANOVA showed a significant main effect for object orientation $(F(2,60)=8.68$, $p=0.0005)$. Participants showed left-sided rotation preferences for $90^{\circ}$ objects $(0.22 \pm 0.44)$ and right-sided rotation preferences for $270^{\circ}$ objects $(-0.13 \pm 0.42)(p=0.0002)$. Critically, a left-sided rotation preference was also observed for $180^{\circ}$ objects $(0.10 \pm 0.46)$; the mean laterality index score did not differ from that for $90^{\circ}$ objects $(p=0.20)$ but from that for $270^{\circ}$ objects $(p=0.006)$.

Laterality index scores for correct trials (see Table 2): Also, this ANOVA showed a significant main effect for object orientation $(\mathrm{F}(2,60)=44.81, p<0.0001)$ with all post hoc comparisons being significant (all $p$-values $<0.0003$ ). The left-sided rotation preference for $90^{\circ}$ objects $(0.52 \pm 0.54)$ and the right-sided rotation preference for $270^{\circ}$ objects $(-0.38 \pm 0.56)$ were more pronounced than that for all trials, but the left-sided preference for $180^{\circ}$ objects was small $(0.12 \pm 0.53)$. The significant interaction between MI groups and object orientation $(\mathrm{F}(2,60)=3.29, p=0.04)$ emerged from differences between the MI groups for $270^{\circ}$ objects; the low MI group revealed a more pronounced rightward rotation bias than the high MI group $(p=0.0005)$, no group differences were found for $90^{\circ}(p=0.84)$ and $180^{\circ}$ $(p=0.26)$ objects.

The findings from Experiment 1 confirmed that objects were preferentially rotated along the shortest trajectory. In addition, participants showed a leftward rotation preference for $180^{\circ}$ objects, which was statistically comparable to the one for $90^{\circ}$ objects. This, together with the observation that rightward rotation preferences for $270^{\circ}$ objects were lesser pronounced than leftward rotation preferences for $90^{\circ}$ objects, points to an overall leftward rotation preference. The different laterality index scores in the high and low MI group for $270^{\circ}$ objects supports our original prediction that stereotyped leftward rotations of high MI scorers would attenuate task performance for objects efficiently to be rotated into the nonpreferred direction. The low MI group adapted efficiently to a rightward rotation preference for $270^{\circ}$ objects and a leftward rotation preference for $90^{\circ}$ objects. Conversely, the high MI group showed a leftward rotation preference for $90^{\circ}$ objects, but only a negligible side preference for $270^{\circ}$ objects. This latter observation indicates that participants rotated $270^{\circ}$ objects more often along the time consuming, inefficient counterclockwise trajectory, not overcoming the stereotyped responding towards the preferred counterclockwise direction. This relative leftward rotation bias in high MI scorers would add further evidence for a relative right-hemispheric hyperdopaminergia in this population (see also Brugger and Graves, 1997; Mohr et al, 2003a). Note that this evidence derives from a task investigating object rotation in the $2 \mathrm{D}$ plane, while the previous studies focused on rotational biases in 3D space.

\section{EXPERIMENT 2}

\section{Participants}

A total of 40 healthy men were recruited by flyers and personal contact. None of them had participated in Experiment 1 . All of them were right-handed according to a 13-item handedness questionnaire (Chapman and 
Chapman, 1987). Again, participants with any current medication, history of drug abuse, or neuropsychiatric illness had been excluded during an extended clinical interview (Campbell, 2000). After a full description of the study, written informed consent was obtained from all participants.

\section{Magical Ideation Scale}

In a procedure identical to Experiment 1, each participant filled in the MI scale prior to experimental testing (Eckblad and Chapman, 1983). In the present experiment, we did not test participants, who scored in the upper quartile of this scale (MI scores $>22$ ), since DA agonists might trigger a psychotic breakdown (Janowsky and Risch, 1979; Sekine et al, 2001), with a presumably elevated risk for participants with high MI scores. The local Ethics Committee had approved the study provided such precautions would be taken. This exclusion was considered unlikely to drastically influence results, since neuropsychological performance patterns described in schizophrenia or healthy schizotypal individuals have even been observed in random samples of completely healthy participants as a function of MI scores (Brugger and Graves, 1997; Mohr et al, 2001, 2003a, b).

\section{Visuo-Motor Rotation Task}

Task and procedure were identical to Experiment 1.

\section{Double-Blind Procedure}

The study was a randomized, double-blind levodopa/ placebo design. A dual-release formulation of levodopa/ benserazide (brand name: Madopar ${ }^{\circledR}$ DR, Roche Pharma (Schweiz) AG, Reinach, Switzerland) with a fast absorption within the first hour and sustained concentration levels thereafter (Gasser et al, 1999) was administered. Prior to the study, participants were informed about the experimental procedure and the possible side effects of levodopa. Each participant fasted overnight and arrived at 9 a.m. on the experimental day. Participants were also instructed not to consume any alcohol or other drugs for at least $24 \mathrm{~h}$ before testing. After having provided informed consent, participants received either Madopar ${ }^{\circledR}$ DR or a placebo. Participants consumed $200 \mathrm{ml}$ water directly after substance administration, and a standardized breakfast was provided 15 min later. In order to insure that participants were under significant levodopa concentration throughout the experiment, two blood samples of about 5-7 ml each were drawn. The first blood sample was collected $30 \mathrm{~min}$ after drug administration. The experiments started afterwards. The first trial of the computer task was conducted about $1 \mathrm{~h}$ after the first blood sample and the second trial about $45 \mathrm{~min}$ after the first trial. As soon as the experiments were finished (about $120 \mathrm{~min}$ after the first blood sample), a second blood sample was drawn.

\section{Blood Sample Collection and Analysis}

The blood was collected in plastic tubes containing lithium heparinate as an anticoagulant and plasma was separated by centrifugation. The samples were stored immediately at $-80^{\circ} \mathrm{C}$ pending analysis. In a first analytical step, to eliminate interfering substances, an internal standard was added to the blood serum samples. Then, the blood serum was fixed on activated alumina, in basic media, and thoroughly vortexed. The liquid phase was discarded and the alumina was finally washed with ultrapure water. Then, levodopa and the internal standard were eluted in acidic media and determined by high-pressure liquid chromatography (HPLC) with electrochemical detection (ECD). The substances were separated on a reversed-phase column and detected by ECD in amperometric mode. Quantification was carried out by internal standard method. Analytical reproducibility was $10 \%$ and quantification limit was $3 \mathrm{ng} / \mathrm{ml}$.

\section{Data Analysis}

Due to an error in the randomization of placebo and levodopa, 21 participants were in the placebo group and 19 participants in the levodopa group. Two participants (one placebo participant and one levodopa participant) rarely made any leftward key presses $(n<10)$, thus disobeying instructions. One placebo participant made use of the leftsided key only seven times (MI score $=3$ ) and one levodopa participant (MI score $=5$ ) never made use of the left-sided key, while the remaining participants made on average 128.1 \pm 58.2 left-sided key presses. They were excluded from further analyses. Moreover, one additional placebo participant $(\mathrm{MI}$ score $=4$ ) was excluded because he performed an overproportionally large number of rightward key presses for $90^{\circ}$ objects $(n=127)$, while the remaining participants made on average only $28.0 \pm 27.5$ rightward key presses for $90^{\circ}$ objects. Since participants scoring in the upper quartile of the MI scales have not been included in this study, the median (6) of the whole group was lower than in Experiment 1. The median split created a high $(n=20$; nine levodopa participants and 11 placebo participants) and low ( $n=17$; nine levodopa participants and eight placebo participants) MI group.

We performed analogous ANOVAs as described for Experiment 1, except that gender was excluded (only men were tested) and substance group (levodopa versus placebo) was added as a between-subject factor. Post hoc single comparisons, if not otherwise stated, were assessed with Duncan's multiple range tests correcting for multiple comparisons. All tests were two-tailed and the $\alpha$-level was set at 0.05 .

\section{RESULTS AND BRIEF DISCUSSION}

\section{Participants}

The three-way ANOVAs with substance groups (placebo vs levodopa) and MI groups (high vs low) as between-subject factors on (1) age and (2) years of education were not significant (all F-values $<1.70, p$-values $>0.20$, Table 3 ). A comparable ANOVA on MI scores ruled out any difference in the substance groups with respect to the MI scores in the two MI groups, since neither the main effect for substance group $(\mathrm{F}(1,33)=1.40, p=0.25)$ nor the interaction between gender and MI group $(\mathrm{F}(1,33)=0.60, p=0.45)$ were significant. 
Table 3 Descriptive Data of the Study Sample in Experiment 2 for the Two Magical Ideation (MI) and Substance Groups Separately

\begin{tabular}{lcccc}
\hline & \multicolumn{2}{c}{ Low MI group } & \multicolumn{1}{c}{ High MI group } \\
\cline { 2 - 4 } & Levodopa group & Placebo group & Levodopa group & Placebo group \\
\hline Ml scores & $3.0 \pm 0.9$ & $3.6 \pm 1.6$ & $9.6 \pm 5.0$ & $11.9 \pm 4.6$ \\
Education (years) & $16.6 \pm 3.0$ & $17.6 \pm 3.1$ & $15.8 \pm 3.5$ & $24.4 \pm 4.9$ \\
Age (years) & $23.9 \pm 3.5$ & $25.9 \pm 3.2$ & $25.7 \pm 3.6$ \\
\hline
\end{tabular}

Table 4 Mean $( \pm S D)$ Laterality Index Score for All Trials (correct+incorrect) for $90^{\circ}$, $180^{\circ}$, and $270^{\circ}$ Rotated Objects and the Two Magical Ideation (MI) and Substance Groups, Separately

\begin{tabular}{|c|c|c|c|c|}
\hline & \multicolumn{2}{|c|}{ Low MI group } & \multicolumn{2}{|c|}{ High MI group } \\
\hline & Levodopa group & Placebo group & Levodopa group & Placebo group \\
\hline $180^{\circ} * * *$ & $-0.02 \pm 0.59$ & $-0.04 \pm 0.68$ & $0.41 \pm 0.45$ & $-0.24 \pm 0.33$ \\
\hline $270^{\circ}$ & $-0.32 \pm 0.56$ & $-0.24 \pm 0.65$ & $-0.41 \pm 0.39$ & $-0.41 \pm 0.33$ \\
\hline
\end{tabular}

Interaction significant $* p<0.001, * * p=0.07$

Table 5 Mean ( \pm SD) Laterality Index Score (Correct Responses) for $90^{\circ}$, $180^{\circ}$, and $270^{\circ}$ Rotated Objects and the Two Magical Ideation (MI) and Substance Groups, Separately

\begin{tabular}{|c|c|c|c|c|}
\hline & \multicolumn{2}{|c|}{ Low MI group } & \multicolumn{2}{|c|}{ High MI group } \\
\hline & Levodopa group & Placebo group & Levodopa group & Placebo group \\
\hline $180^{\circ} * * *$ & $-0.03 \pm 0.62$ & $-0.02 \pm 0.67$ & $0.41 \pm 0.45$ & $-0.23 \pm 0.34$ \\
\hline $270^{\circ}$ & $-0.59 \pm 0.45$ & $-0.64 \pm 0.51$ & $-0.76 \pm 0.20$ & $-0.72 \pm 0.23$ \\
\hline
\end{tabular}

Interaction significant $* p=0.006, * * * 0.07$

In the levodopa group, the mean levodopa serum concentration was $213.5 \pm 232.6 \mathrm{ng} / \mathrm{ml}$ for the first blood sample and $142.2 \pm 158.5 \mathrm{ng} / \mathrm{ml}$ for the second blood sample $\left(t_{17}=1.23, p=0.24\right)$. None of the participants reported any remarkable substance effect. Levodopa serum concentrations in the placebo group were zero, throughout.

\section{Visuo-Motor Rotation Task}

Laterality index scores for all trials, irrespective of response correctness (see Table 4): The ANOVA revealed a significant main effect for object orientation $(\mathrm{F}(2,66)=39.01$, $p<0.0001)$. Post hoc comparisons showed significant differences between objects (all $p$-values $<0.0002$ ) with a pronounced leftward rotation preference for $90^{\circ}$ objects $(0.36 \pm 0.53)$ and rightward preference for $270^{\circ}$ objects $(-0.38 \pm 0.47)$ as well as almost no side preference for $180^{\circ}$ objects $(0.02 \pm 0.55)$. The interaction between MI groups and substance groups was significant $(\mathrm{F}(1,33)=4.99$, $p=0.03$ ) indicating that high MI participants showed a rightward rotation preference in the placebo group
$(-0.22 \pm 0.29)$, but a leftward rotation preference in the levodopa group $(0.22 \pm 0.27, p=0.03)$. Single comparisons with respect to the low MI groups were not significant (placebo group: $0.08 \pm 0.45$; levodopa group: $-0.04 \pm 0.48$; all $p$-values $>0.11)$. The three-way interaction was also significant $(\mathrm{F}(2,66)=5.52, p=0.006)$. We performed three ANOVAs on each laterality index score separately. The interaction between MI groups and substance groups in the ANOVA on $90^{\circ}$ objects was significant $(\mathrm{F}(1,33)=13.32$, $p=0.0009)$. The high MI group had a stronger leftward rotation bias in the levodopa than (1) placebo group $(p=0.005)$ and (2) low MI participants in the levodopa group $(p=0.05)$. In the placebo group, the low MI group had a stronger leftward rotation preference than the high MI group $(p=0.006)$. A somewhat similar picture was observed for the ANOVA on $180^{\circ}$ objects. Although the interaction was only a statistical trend $(\mathrm{F}(1,33)=3.45, p=0.07)$, the high MI group's leftward rotation bias was stronger in the levodopa group than its rightward rotation bias in the placebo group $(p=0.02)$. The ANOVA on $270^{\circ}$ objects was not significant (all F-values $<0.20$ ). 


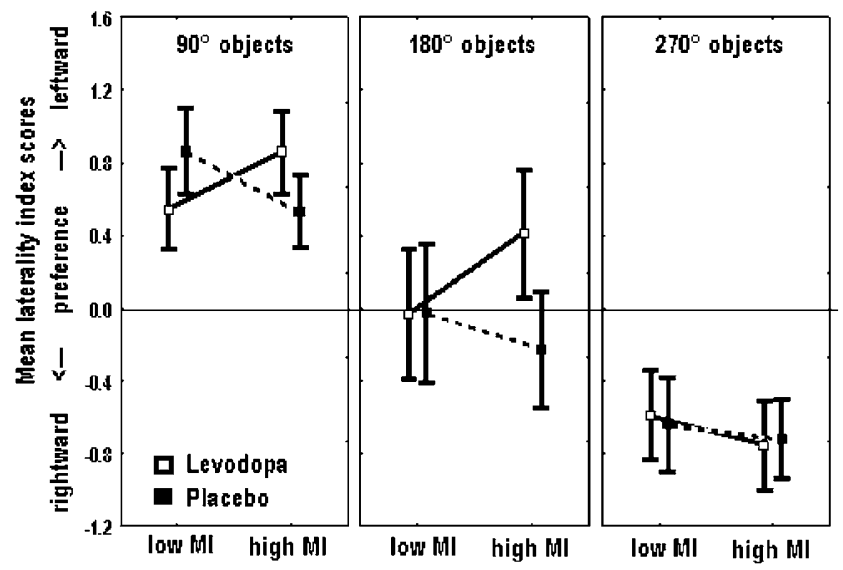

Figure 2 Mean laterality index scores for correct trials in the two substance groups (dashed line placebo group, black line levodopa group). Performance is depicted for the high and low MI groups and the three object types separately. Vertical bars denote 0.95 confidence intervals.

Laterality index scores on correct trials (see Table 5): The ANOVA again showed a significant main effect for stimulus orientation $(\mathrm{F}(2,66)=168.02, p<0.0001)$ with all post hoc comparisons being significant ( $p$-values $<0.0002)$. The three-way interaction was again significant $(\mathrm{F}(2,66)=3.91$, $p=0.02)$. As for the ANOVA on all trials, we conducted three separate ANOVAs on the laterality index scores separately. The interaction for $90^{\circ}$ objects was significant $(\mathrm{F}(1,33)=8.67, p=0.006$, Figure 2$)$. The leftward rotation bias was (1) stronger in high MI participants in the levodopa group relative to that of high MI participants in the placebo group $(p=0.05)$; and (2) in the high MI levodopa participants than in the low MI levodopa participants $(p=0.05)$. In the placebo group, the leftward rotation bias was stronger in the low than high MI group $(p=0.05)$. A somewhat similar picture was observed for the ANOVA on $180^{\circ}$ objects (Figure 2). Although the interaction was only a statistical trend $(\mathrm{F}(1,33)=3.49, p=0.07)$; the high MI group's leftward rotation preference was stronger in the levodopa group than its rightward bias in the placebo group $(p=0.02)$. The ANOVA on $270^{\circ}$ objects did not produce any significant main effects nor interactions (all F-values $<1.10$, Figure 2).

As in the participants of Experiment 1, rotational preferences occurred preferentially along the shortest trajectory. However, the patterns of rotational biases as a function of participants' MI scores were quite different in the present sample (placebo group) from the population tested in Experiment 1. We neither replicated a general leftward rotation preference in the placebo group nor did we find a more pronounced leftward rotation bias in the high compared to the low MI group. On the contrary, high MI scorers revealed a rightward rotation bias in the placebo group, but a leftward rotation bias in the levodopa group. Moreover, in the placebo group, low MI scorers had a stronger leftward rotation bias than high MI scorers. The opposite rotational biases in the high MI groups clearly speak against our original idea that high MI scores would generally relate to leftward orientation bias for object rotations in the $2 \mathrm{D}$ plane. Thus, we have to reconsider our initial assumption that a relative right-hemispheric hyperdopaminergia in high MI scorers might lead to stereotyped leftward rotation preferences, at least when rotational preferences are assessed for object rotations in the 2D plane. The absence of a consistent rotational bias towards one side in the present visuo-motor task would also highlight the need to determine more precisely those 'turning' tasks and related experimental parameters, which reportedly are under dopaminergic mediation (Mohr et al, 2004). As a further consequence, we are reluctant to interpret the observed leftward rotation preference in high MI scorers in the levodopa group as a result of a substanceinduced right-hemispheric hyperdopaminergia.

Having listed the critical points that emerged from the apparently divergent findings between the two experiments, we now point to one interesting pattern, common to Experiments 1 and 2 (placebo group). High MI scorers responded more stereotyped than did low MI scores. In the placebo group of Experiment 2, the high MI group showed a stereotyped rightward rotation bias resulting in an efficient rightward preference for $270^{\circ}$ objects, a rightward rotation preference for $180^{\circ}$ objects, and an inefficient laterality index score around zero for $90^{\circ}$ objects (all trials). The laterality index score for $90^{\circ}$ objects on correct trials was positive, indicating that high MI participants performed many inefficient rightward rotations for $90^{\circ}$ objects leading to many incorrect trials. Thus, high MI scorers' response stereotypy consisted of a rotation bias in a rightward direction. This conclusion is also supported by the findings that these high MI scorers showed (1) a pronounced rightward bias for $180^{\circ}$ objects (which was around zero for low MI scorers) and (2) a stronger rightward bias than low MI scorers for $270^{\circ}$ objects. Taking these results and those from Experiment 1 together, we conjecture that, whatever the direction of the rotational bias may be in a given sample, high MI scorers perform more stereotyped than low MI scorers. This makes them less efficient in swiftly adopting different rotation strategies in response to current task demands.

However, high MI scorers did not respond even more stereotyped in the levodopa than in the placebo group. Rather, they showed a more sophisticated rotation strategy than did the low MI scorers. Despite a general leftward rotation bias in the levodopa group, high MI scorers showed a pronounced rightward bias for $270^{\circ}$ objects (Tables 4 and 5). Low MI scorers, on the other hand, showed less of a leftward rotation bias in the levodopa compared to the placebo group. This was particularly evident for $90^{\circ}$ objects, for which a leftward bias is the optimal rotation strategy. These results suggest that elevated DA concentrations do not increase stereotyped responding in high MI scorers, but result in most efficient task performance. In contrast, low MI scorers' performance, if influenced at all by levodopa treatment, appeared to deteriorate (Figure 2).

\section{General Discussion}

As reviewed in the introduction, stereotyped spatial and nonspatial responding has been described for patients with schizophrenia, schizotypal personality disorder, healthy participants showing schizotypal features, and healthy participants after the administration of DA agonists. We tested healthy participants' stereotyped responding in clockwise and counterclockwise directions in a computer 
task resembling the popular TETRIS computer game. Within $2 \mathrm{~s}$, 'dropping' line drawings had to be rotated into a target orientation, indicated by a second object depicted at the bottom of the computer screen. We predicted more stereotyped responding for high compared to low scorers on the MI scale (Eckblad and Chapman, 1983). This prediction was born out in Experiment 1 and in the placebo group of Experiment 2. Opposite to our expectancies, in the levodopa group of Experiment 2, high MI scorers did not yield even more stereotyped responding, but performed the task in a most efficient and adaptive way.

Successful performance in our visuo-motor rotation task depends on complex attentional and executive functions (see also Haier et al, 1992). Results from previous studies would suggest that functions of the left hemisphere play a more important role for these functions than those of the right hemisphere (Haier et al, 1992; Koepp et al, 1998). Haier et al (1992), for instance, showed that novices' performance in the TETRIS game was related to increased cerebral blood flow in the supramarginal gyrus and lateral occipital areas of the left hemisphere. Another PET study reported enhanced endogenous striatal DA release, in particular in the left hemisphere, during a goal-directed visuo-motor computer game (Koepp et al, 1998). Selectively impaired left hemisphere functioning has been reported for healthy participants with elevated positive schizotypal features (Mohr et al, 2001; Park, 1999) and unmedicated patients with schizophrenia (Purdon and Flor-Henry, 2000). Thus, the inferior rotation strategy in high MI scorers might be yet another example for a relative impairment of primarily left-hemisphere mediated functions.

However, such impairment does not explain the efficient rotation strategy observed in high MI scorers in the levodopa group. Originally, we expected that performance would deteriorate under a DA agonist given that (1) DA agonists can worsen psychotic symptoms in patients with schizophrenia (Davidson et al, 1987; Abi-Dargham et al, 1998) and trigger psychotic symptoms in healthy participants (Janowsky and Risch, 1979; Sekine et al, 2001); (2) schizotypy and schizophrenia share cognitive (Gooding et al, 1999; Park, 1999), attentional (Sarkin et al, 1998; Mohr et al, 2003a), behavioral (Barnett and Corballis, 2002; Mohr et al, 2003b), and physiological (Klein et al, 1999; Pizzagalli et al, 2000) similarities; and (3) DA agonists increase stereotyped responding in animals (Randrup and Munkvad, 1974; Staton and Solomon, 1984; Kelley et al, 1988) and healthy populations (Connell, 1958; Ridley et al, 1988). Findings from animal (Arnsten, 1997; Williams and Goldman-Rakic, 1995) and human (Mehta et al, 2000) studies propose that dopaminergic actions follow an inverted Ushape function, with an improvement of cognitive performance from low to medium, but deterioration from medium to high doses. This characteristic inverted U-shape function is thought to explain DA actions on cognition as a function of individuals' overall baseline performance. Animals (Granon et al, 2000) and humans (Kimberg et al, 1997; Mattay et al, 2000; Mehta et al, 2000) performing low at baseline profited from the substance and those performing high at baseline deteriorated after substance intake. Thus, applied to the present study, individuals performing low in the substance-free state (high MI scorers in Experiment 1 and the placebo group of Experiment (2) performed best in the levodopa group. On the other hand, individuals performing high in a substance-free state (low MI scores in Experiment 1 and the placebo group of Experiment 2) performed similar or even worse in the levodopa group. ${ }^{1}$

Whether targeting behavior or pharmacology, most research so far focused on similarities rather than on dissimilarities between schizophrenia and schizotypy. If similarities between schizotypy and schizophrenia existed on the neurochemical level, one would expect task performance to improve in schizotypal individuals with (second-generation) DA antagonists. Specifically, atypical neuroleptic agents were found to improve neuropsychological functioning including immediate recall, visuo-spatial construction, executive functions, verbal fluency, attention, and working memory (see eg Cuesta et al, 2001; Meltzer and McGurk, 1999; Purdon et al, 2001; Sumiyoshi et al, 2003 for overviews). However, our schizotypal participants performed in a most adaptive way after a DA agonist. This dissociation would point to neurochemical differences between schizotypy and schizophrenia. It could be argued that such differences emerge from a pathological 'hypofrontality' in patients with schizophrenia (Andreasen et al, 1992). In fact, stereotyped and/or perseverative behavior in schizophrenia is not necessarily related to a generally hyperactive DA system but to a selective hypofunctioning within the frontal lobes (Perry and Braff, 1998). It was thus challenging to propose that a hypofunctional frontal system in schizophrenia might improve with DA agonist treatment, which, in fact, was the case, but only (1) when patients were prior stabilized with DA antagonists (Daniel et al, 1991; Szeszko et al, 1999), and (2) DA activity was kept on an intermediate level (Szeszko et al, 1999).

In conclusion, we showed that high positive schizotypy is associated with stereotyped behavior in a visuo-motor computer task. After levodopa administration, however, stereotyped behavior was absent in the high MI scorers, and task strategy was even superior to that of low MI participants. We conjecture that healthy participants with high MI scores are able to compensate a single DA challenge rather than to further deteriorate. For populations with a

\footnotetext{
1 Two anonymous reviewers pointed to the relevance of gender or deficitlike or negative symptoms to the dopamine-mediated findings of the present two experiments (see also Siever and Davis, 2004 for overview). Female participants were tested in Experiment 1, but only men were tested in Experiment 2. Moreover, deficit-like symptoms might be more prevailing in female participants or in participants with high MI scores in one but not the other substance group. Thus, our results might be rather a result of different distributions of negative schizotypal features in the two MI groups in (1) women and men (Experiment 1 or 2) in the levodopa and placebo group (Experiment 2). We assessed participants' scores on the physical anhedonia scale (Chapman et al 1976, revised German version Meyer and Hautzinger, 1999). An ANOVA with MI groups and gender as betweensubject measures (Experiment 1) on physical anhedonia scores did not show a significant interaction $(\mathrm{F}(1,30)=1.14 ; 0.29)$. Likewise, an ANOVA with MI groups and substance groups as between-subject measures (Experiment 2) on physical anhedonia did not show a significant interaction $(\mathrm{F}(1,33)=0.12, p=0.73)$. Thus, we assume that deficit-like symptoms, among which physical anhedonia is a key feature, were not the major determinant for the findings observed in the present study. However, future studies would profit from a more careful assessment of different symptom dimensions.
} 
diagnosis of schizotypal personality disorder, accumulated evidence for intact compensatory and/or protective brain mechanisms against full-blown psychosis has recently be discussed (Siever and Davis, 2004). Moreover, Siegel et al (1996) showed that DA agonistic treatment in schizotypal personality disorder reduced perseverative errors in the Wisconsin Card Sorting Test. Wuebben and Winterer (2001) found frontally pronounced resting EEG activity in patients with schizophrenia but not healthy schizotypal participants. Thus, our present findings would extend the observations of potential protective brain mechanisms along the schizophrenia spectrum including healthy schizo typal populations. In addition to these considerations, such protective mechanisms may explain why even large longitudinal studies on participants with high MI scores, as undertaken by the Chapman group (Chapman et al, 1994; Kwapil et al, 1997), failed to convincingly predict a later psychotic breakdown from elevated positive schizotypal features alone (see also Verdoux and van Os, 2002). Indeed, high MI might also predict a later mood disorder (Chapman et al, 1994) and is a common feature not only of the schizophrenia spectrum disorders but also a symptom of some personality disorders (eg Lyons et al, 1995; Kwapil et al, 1997). Thus, positive schizotypy may be accompanied by a relative hyperdopaminergia, but yet reflects just one of the many DA-mediated variations of the human mind and personality (Horrobin, 1998; Previc, 1999) accompanied by distinct cognitive capacities (Gianotti et al, 2001; Weinstein and Graves, 2002) and, ultimately, an unusual way of perceiving the world (Wolfradt et al, 1999; Stöckenius and Brugger, 2000; McCreery and Claridge, 2002; Van de Ven and Merckelbach, 2003).

\section{ACKNOWLEDGEMENTS}

This work was supported in part by Grant \#690610 from the Institut für Grenzgebiete der Psychologie und Psychohygiene, Freiburg i. Brsg, Germany and by Grant \#3165096.01 from the Swiss National Science Foundation. We thank Marianne Wackermann for blood serum preparation, Lotti Batschelet and the nurses on floor C of the Department of Neurology at the University Hospital of Zurich for blood sample collection. Last, but not least, without Martina Siemann's (Konstanz, Germany) programming skills and her insightful comments, the present study would not have been possible.

\section{REFERENCES}

Abi-Dargham A, Gil R, Krystal J, Baldwin RM, Seibyl JP, Bowers M et al (1998). Increased striatal dopamine transmission in schizophrenia: confirmation in a second cohort. Am J Psychiatry 155: 761-767.

Andreasen NC, Rezai K, Alliger R, Swayze VW II , Flaum M, Kirchner $\mathrm{P}$ et al (1992). Hypofrontality in neuroleptic-naive patients and in patients with chronic schizophrenia. Assessment with xenon 133 single-photon emission computed tomography and the Tower of London. Arch Gen Psychiatry 49: 943-958.

Arnsten AF (1997). Catecholamine regulation of the prefrontal cortex. J Psychopharmacol 11: 151-162.
Avons SE, Nunn JA, Chan L, Armstrong H (2003). Executive function assessed by memory updating and random generation in schizotypal individuals. Psychiatry Res 120: 145-154.

Barnett KJ, Corballis MC (2002). Ambidexterity and magical ideation. Laterality 7: 75-84.

Bleuler E (1911/1950). Dementia Praecox or the Group of the Schizophrenias. International University Press: New York.

Bracha HS, Livingston RL, Clothier J, Linington BB, Karson CN (1993). Correlation of severity of psychiatric patients' delusions with right hemispatial inattention (left-turning behavior). Am J Psychiatry 150: 330-332.

Bracha HS, Shults C, Glick SD, Kleinman JE (1987). Spontaneous asymmetric circling behavior in hemi-parkinsonism: a human equivalent of the lesioned-circling rodent behavior. Life Sci 40: 1127-1130.

Brugger P (1997). Variables that influence the generation of random sequences: an update. Percept Mot Skills 84: 627-661.

Brugger P, Graves RE (1997). Right hemispatial inattention and magical ideation. Eur Arch Psychiatry Clin Neurosci 247: 55-57.

Brugger P, Landis T, Regard M (1990). A 'sheep-goat effect' in repetition avoidance: extrasensory perception as an effect of subjective probability? Br J Psychol 81: 455-468.

Cadenhead KS, Perry W, Shafer K, Braff DL (1999). Cognitive functions in schizotypal personality disorder. Schizophr Res 37: 123-132.

Campbell JJ (2000). Neuropsychiatric assessment. In: Coffey CE, Cummings JL (eds). Textbook of Geriatric Neuropsychiatry, 2nd edn. American Psychiatric Press: Washington, DC. pp 109-124.

Chapman LJ, Chapman JP (1987). The measurement of handedness. Brain Cogn 6: 175-183.

Chapman LJ, Chapman JP, Kwapil TR, Eckblad M, Zinser MC (1994). Putatively psychosis-prone subjects 10 years later. J Abnorm Psychol 103: 171-183.

Chapman LJ, Chapman JP, Raulin ML (1976). Scales for physical and social anhedonia. J Abnorm Psychol 85: 374-382.

Connell PH (1958). Amphetamine Psychosis, Maudsley Monographs No. 5. Oxford University Press: Oxford.

Cuesta MJ, Peralta V, Zarzuela A (2001). Effects of olanzapine and other antipsychotics on cognitive function in chronic schizophrenia: a longitudinal study. Schizophr Res 48: 17-28.

Daniel DG, Weinberger DR, Jones DW, Zigun JR, Coppola R, Handel S et al (1991). The effect of amphetamine on regional cerebral blood flow during cognitive activation in schizophrenia. J Neurosci 11: 1907-1917.

Davidson M, Davis KL (1988). A comparison of plasma homovanillic acid concentration in schizophrenic patients and normal controls. Arch Gen Psychiatry 45: 561-563.

Davidson M, Keefe RS, Mohs RC, Siever LJ, Losonczy MF, Horvath TB et al (1987). L-dopa challenge and relapse in schizophrenia. Am J Psychiatry 144: 934-938.

Davis KL, Kahn RS, Ko G, Davidson M (1991). Dopamine in schizophrenia: a review and reconceptualization. Am J Psychiatry 148: 1474-1486.

Eckblad M, Chapman LJ (1983). Magical ideation as an indicator of schizotypy. J Consult Clin Psychol 51: 215-225.

Garety P, Wessely S (1994). The assessment of positive symptoms In: Barnes TRE, Nelson HE (eds). The Assessment of Psychoses, A Practical Handbook. Chapman \& Hall: London. pp 21-39.

Garner JP, Meehan CL, Mench JA (2003). Stereotypies in caged parrots, schizophrenia and autism: evidence for a common mechanism. Behav Brain Res 145: 125-134.

Gasser UE, Jorga K, Crevoisier C, Hovens SE, van Giersbergen PL (1999). COMT inhibition by tolcapone further improves levodopa pharmacokinetics when combined with a dual-release formulation of levodopa/benserazide. A novel principle in the treatment of Parkinson's disease. Eur Neurol 41: 206-211. 
Gianotti LR, Mohr C, Pizzagalli D, Lehmann D, Brugger P (2001). Associative processing and paranormal belief. Psychiatry Clin Neurosci 55: 595-603.

Gooding DC, Kwapil TR, Tallent KA (1999). Wisconsin Card Sorting Test deficits in schizotypic individuals. Schizophr Res 40: 201-209.

Granon S, Passetti F, Thomas KL, Dalley JW, Everitt BJ, Robbins TW (2000). Enhanced and impaired attentional performance after infusion of $\mathrm{D} 1$ dopaminergic receptor agents into rat prefrontal cortex. J Neurosci 20: 1208-1215.

Haier RJ, Siegel Jr BV, MacLachlan A, Soderling E, Lottenberg S, Buchsbaum MS (1992). Regional glucose metabolic changes after learning a complex visuospatial/motor task: a positron emission tomographic study. Brain Res 570: 134-143.

Harvey PD, Green MF, McGurk SR, Meltzer HY (2003). Changes in cognitive functioning with risperidone and olanzapine treatment: a large-scale, double-blind, randomized study. Psychopharmacology 169: 404-411.

Horrobin DF (1998). Schizophrenia: the illness that made us human. Med Hypotheses 50: 269-288.

Janowsky DS, Risch C (1979). Amphetamine psychosis and psychotic symptoms. Psychopharmacology 65: 73-77.

Jones IH (1965). Observation of schizophrenic stereotypies. Compr Psychiatry 6: 323-335.

Kelley AE, Lang CG, Gauthier AM (1988). Induction of oral stereotypy following amphetamine microinjection into a discrete subregion of the striatum. Psychopharmacology 95: 556-559.

Kimberg DY, D'Esposito M, Farah MJ (1997). Effects of bromocriptine on human subjects depend on working memory capacity. Neuroreport 8: 3581-3585.

Klein C, Berg P, Rockstroh B, Andresen B (1999). Topography of the auditory P300 in schizotypal personality. Biol Psychiatry 45: $1612-1621$.

Koepp MJ, Gunn RN, Lawrence AD, Cunningham VJ, Dagher A, Jones $\mathrm{T}$ et al (1998). Evidence for striatal dopamine release during a video game. Nature 393: 266-268.

Kraeplin E (1919) Dementia praecox and paraphrenia (transl. RM Barclay) RE Kreiger: New York. (reprinted 1971).

Kuczenski R, Segal DS (1999). Sensitization of amphetamineinduced stereotyped behaviors during the acute response. $J$ Pharmacol Exp Ther 288: 699-709.

Kwapil T, Miller M, Zinser M, Chapman J, Chapman L (1997). Magical ideation and social anhedonia as predictors of psychosis proneness: a partial replication. J Abnorm Psychol 106: 491-495.

Laruelle M, Abi-Dargham A (1999). Dopamine as the wind of the psychotic fire: new evidence from brain imaging studies. $J$ Psychopharmacol 13: 358-371.

Lenzenweger MF, Korfine L (1994). Perceptual aberrations, schizotypy, and the Wisconsin Card Sorting Test. Schizophr Bull 20: 345-357.

Levine J, Martine T, Feraro R, Kimhi R, Bracha HS (1997). Medicated chronic schizophrenic patients do not demonstrate left turning asymmetry. Neuropsychobiology 36: 22-24.

Lyons MJ, Toomey R, Faraone SV, Kremen WS, Yeung AS, Tsuang MT (1995). Correlates of psychosis proneness in relatives of schizophrenic patients. J Abnorm Psychol 104: 390-394.

Marshall JC, Caplan D, Holmes JM (1975). The measure of laterality. Neuropsychologia 13: 315-321.

Mattay VS, Callicott JH, Bertolino A, Heaton I, Frank JA, Coppola $\mathrm{R}$ et al (2000). Effects of dextroamphetamine on cognitive performance and cortical activation. Neuroimage 12: 268-275.

McCreery C, Claridge G (2002). Healthy schizotypy: the case of out-of-the-body experiences. Pers Indiv Diff 32: 141-154.

Mehta MA, Owen AM, Sahakian BJ, Mavaddat N, Pickard JD, Robbins TW (2000). Methylphenidate enhances working memory by modulating discrete frontal and parietal lobe regions in the human brain. J Neurosci 20: RC65.
Meltzer HY, McGurk SR (1999). The effects of clozapine, risperidone, and olanzapine on cognitive function in schizophrenia. Schizophr Bull 25: 233-255.

Meyer TD, Hautzinger M (1999). Two year stability of psychosis proneness scales and their relationship to personality disorder traits. J Pers Assess 73: 472-488.

Mohr C, Bracha HS, Brugger P (2003a). Magical ideation modulates spatial behavior. J Neuropsychiatry Clin Neurosci 15: 168-174.

Mohr C, Brugger P, Bracha HS, Landis T, Viaud-Delmon I (2004). Human side preferences in three different whole-body movement tasks. Behav Brain Res 151: 321-326.

Mohr C, Rőhrenbach CM, Laska M, Brugger P (2001). Unilateral olfactory perception and magical ideation. Schizophr Res 47: 255-264.

Mohr C, Thut G, Landis T, Brugger P (2003b). Hands, arms, and minds: interactions between posture and thought. J Clin Exp Neuropsychol 25: 1000-1010.

Park S (1999). Hemispheric asymmetry of spatial working memory deficit in schizophrenia. Int J Psychophysiol 34: 313-322.

Peralta V, Cuesta MJ (2001). Motor features in psychotic disorders. I. Factor structure and clinical correlates. Schizophr Res 47: 107116.

Perry W, Braff DL (1998). A multimethod approach to assessing perseverations in schizophrenia patients. Schizophr Res 33: 6977.

Pickar D, Labarca R, Linnoila M, Roy A, Hommer D, Everett D et al (1984). Neuroleptic-induced decrease in plasma homovanillic acid and antipsychotic activity in schizophrenic patients. Science 225: 954-957.

Pizzagalli D, Lehmann D, Gianotti L, Koenig T, Tanaka H, Wackermann J et al (2000). Brain electric correlates of strong belief in paranormal phenomena: intracerebral EEG source and regional Omega complexity analyses. Psychiatry Res 100: 139-154.

Previc FH (1999). Dopamine and the origins of human intelligence. Brain Cogn 41: 299-350.

Purdon SE, Flor-Henry P (2000). Asymmetrical olfactory acuity and neuroleptic treatment in schizophrenia. Schizophr Res 44: 221-232.

Purdon SE, Malla A, Labelle A, Lit W (2001). Neuropsychological change in patients with schizophrenia after treatment with quetiapine or haloperidol. J Psychiatry Neurosci 26: 137-149.

Pycock CJ (1983). Experimental model of hemi-Parkinsonism In: Myslobodsky MS (ed). Hemisyndromes: Psychobiology, Neurology, Psychiatry. Academic Press: New York. pp 69-90.

Raine A, Sheard C, Reynolds GP, Lencz T (1992). Pre-frontal structural and functional deficits associated with individual differences in schizotypal personality. Schizophr Res 7: 237-247.

Randrup A, Munkvad I (1974). Pharmacology and physiology of stereotyped behaviour. J Psychiatric Res 11: 1-10.

Ridley RM, Baker HF, Frith CD, Dowdy J, Crow TJ (1988). Stereotyped responding on a two-choice guessing task by marmosets and humans treated with amphetamine. Psychopharmacology 19: 560-564.

Salamé P, Danion JM, Peretti S, Cuervo C (1998). The state of functioning of working memory in schizophrenia. Schizophr Res 30: $11-29$.

Sarkin AJ, Dionisio DP, Hillix WA, Granholm E (1998). Positive and negative schizotypal symptoms relate to different aspects of crossover reaction time task performance. Psychiatry Res 81: 241-249.

Sekine Y, Iyo M, Ouchi Y, Matsunaga T, Tsukada H, Okada $\mathrm{H}$ et al (2001). Methamphetamine-related psychiatric symptoms and reduced brain dopamine transporters studied with PET. Am J Psychiatry 158: 1206-1214.

Siegel Jr BV, Trestman RL, O'Flaithbheartaigh S, Mitropoulou V, Amin F, Kirrane R et al (1996). D-amphetamine challenge effects 
on Wisconsin Card Sort Test. Performance in schizotypal personality disorder. Schizophr Res 20: 29-32.

Siever LJ, Amin F, Coccaro EF, Bernstein D, Kavoussi RJ, Kalus O et al (1991). Plasma homovanillic acid in schizotypal personality disorder. Am J Psychiatry 148: 1246-1248.

Siever LJ, Amin F, Coccaro EF, Trestman R, Silverman J, Horvath TB et al (1993). CSF homovanillic acid in schizotypal personality disorder. Am J Psychiatry 150: 149-151.

Siever LJ, Davis KL (2004). The pathophysiology of schizophrenia disorders: perspectives from the spectrum. Am J Psychiatry 161: 398-413.

Staton DA, Solomon PR (1984). Microinjections of d-amphetamine into the nucleus accumbens and caudate-putamen differentially affect stereotypy and locomotion in the rat. Physiol Psychol 12: 159-162.

Stőckenius S, Brugger P (2000). Perceived electrosensitivity and magical ideation. Percept Mot Skills 90: 899-900.

Sumiyoshi T, Jayathilake K, Meltzer HY (2003). The effect of melperone, an atypical antipsychotic drug, on cognitive function in schizophrenia. Schizophr Res 59: 7-16.
Szeszko PR, Bilder RM, Dunlop JA, Walder DJ, Lieberman JA (1999). Longitudinal assessment of methylphenidate effects on oral word production and symptoms in first-episode schizophrenia at acute and stabilized phases. Biol Psychiatry 45: 680-686.

Van de Ven V, Merckelbach H (2003). The role of schizotypy, mental imagery, and fantasy proneness in hallucinatory reports of undergraduate students. Pers Indiv Diff 35: 889-896.

Verdoux H, van Os J (2002). Psychotic symptoms in non-clinical populations and the continuum of psychosis. Schizophrenia Res 54: 59-65.

Weinstein S, Graves RE (2002). Are creativity and schizotypy products of a right hemisphere bias? Brain Cogn 49: 138-151.

Williams GV, Goldman-Rakic PS (1995). Modulation of memoryfields by dopamine D1 receptors in prefrontal cortex. Nature 376: $572-575$.

Wolfradt U, Oubaid V, Straube ER, Bischoff N, Mischo J (1999). Thinking styles, schizotypal traits and anomalous experiences. Pers Indiv Diff 27: 821-830.

Wuebben Y, Winterer G (2001). Hypofrontality-a risk-marker related to schizophrenia? Schizophr Res 48: 207-217. 